\title{
Małgorzata Czarnecka
}

Universität Wrocław, Wrocław

DOI: $10.19195 / 0435-5865.141 .12$

\section{„How many elephants?"1 - Probleme mit der Begriffsbestimmung und Identifizierung formelhafter Äußerungen}

\section{Einleitung: Was sind formelhafte Äußerungen?}

In der letzten Zeit ist in der sprachwissenschaftlichen Forschung das Interesse an komplexen sprachlichen Einheiten, die häufig als „formelhaft" bezeichnet werden, erheblich gestiegen. Dieses Phänomen ist im Kontext der Entwicklung der Konstruktionsgrammatik zu sehen. Aus ihrer gebrauchsgestützten Perspektive wird betont, dass (Tomasello 2006: 21)

[...] sich Menschen zu bestimmten Anlässen mit bestimmten, aus konkreten sprachlichen Bausteinen bestehenden Äußerungen verständigen. Wenn Menschen wiederholt ,ähnliche“ Dinge in ,ähnlichen“ Situationen sagen, entwickelt sich daraus mit der Zeit ein sprachliches Verwendungsmuster, das in den Köpfen der Benutzer als neue Kategorie oder Konstruktion schematisiert wird - mit unterschiedlichen Abstraktionsgraden.

Die Sprachstruktur ergibt sich aus dem Sprachgebrauch und die Grammatik einer Sprache ist ein Inventar von Konstruktionen (Fischer / Stefanowitsch 2006: 3). Des Weiteren wird der Spracherwerb als kontextuell gesteuerter Erwerb von unterschiedlich komplexen und abstrakten sprachlichen Mustern dargestellt, und die Entwicklung von Grammatik als lexikalisch-syntaktisches Kontinuum (vgl. Roche / Reher / Simic 2012: 27). Dies hat zur Folge, dass die Nachahmung heutzutage wieder zu einem zentralen Begriff wurde, nachdem sie seit den 1950-er Jahren durch Chomsky's Auseinandersetzung mit dem Paradigma des Behaviorismus aufgegeben worden war (vgl. Leiss 2009: 17).

${ }^{1}$ Dieser Ausdruck wird von Alison Wray im Hinblick auf die in der Literatur herrschende terminologische Uneinheitlichkeit und konzeptuelle Unschärfe formelhafter Äußerungen (als Kapiteltitel) verwendet (Wray 2012: 237). 
Formelhafte Äußerungen sind komplexe sprachliche Einheiten, die dadurch charakteristisch sind, dass sie vom Sprecher flüssig und mühelos ausgeführt werden. Es sind beispielsweise kommunikative Formeln (Guten Tag!, Bis bald!), Kollokationen (reiner Zufall), Idiome, Zitate (Die Würfel sind gefallen); einen formelhaften Charakter haben auch Textteile und ganze Texte (z.B. Abzählreime oder Gebete). In der Forschungsliteratur gibt es weder einen einheitlichen Begriff noch eine einheitliche Definition formelhafter Äußerungen, was auf die Unterschiedlichkeit der Perspektiven und auf die Differenzierung der wissenschaftlichen Disziplinen, die sich dem Thema widmen, zurückzuführen ist (vgl. Czarnecka 2011: 190 ff.). Da es jedoch Elemente gibt, die in den meisten Definitionen zu finden sind, kann angenommen werden, dass sich formelhafte Äußerungen durch folgende Merkmale auszeichnen: Ganzheitlichkeit (sie werden vom Gedächtnis als Ganzes gespeichert und abgerufen), Häufigkeit des Auftretens (sie werden relativ häufig gebraucht) und Situationsgebundenheit (sie werden in spezifischen Situationen verwendet). Dabei ist hervorzuheben, dass eigentlich nur die Ganzheitlichkeit als ausschlaggebend angesehen werden kann (zur Diskussion dazu s. Czarnecka 2010: 35 ff.). All die Merkmale sind miteinander verbunden: da formelhafte Äußerungen ganzheitlich gespeichert sind, werden sie flüssig, korrekt, mühelos und mit einer hohen Geschwindigkeit produziert, was natürlicherweise nicht ohne Zusammenhang mit der Häufigkeit ihres Auftretens stehen kann. Die Häufigkeit kann wiederum mit der Situationsgebundenheit in Zusammenhang gebracht werden; da die Sprache eine Form sozialen Verhaltens ist, so werden die sich ständig wiederholenden konventionalisierten Äußerungen in häufig wiederkehrenden Alltagssituationen von den Sprechern verwendet. Die Angehörigen einer Sprachgemeinschaft machen so oft wie möglich Gebrauch von formelhaften Äußerungen, was mit dem Prinzip der sprachlichen Ökonomie verbunden ist, aber auch im kulturellen und sozialen Kontext gesehen werden soll: „Auf Formelhaftes zurückzugreifen kann als Berufung auf eine Gemeinschaftskonvention verstanden werden, da in der sprachlich festen Form immer auch sprachgemeinschaftlich etablierte kulturelle Erfahrungen stecken, die sich in Formelgestalt in kognitiv einprägsamer Weise konservieren lassen" (Stein 1995: 14-15).

Aufgrund der erwähnten Uneinheitlichkeit des Begriffs wirft sowohl die Begriffsbestimmung als auch die Identifizierung von formelhaften Äußerungen verschiedene Probleme auf. Ziel dieses Beitrags ist es daher, die damit verbundenen Probleme zu skizzieren und die Perspektiven ihrer Lösung aufzuzeigen.

\section{Zur Problematik der Begriffswahl}

Die Wahl eines Begriffes (und der Begriffsdefinition) für die eigenen Forschungsziele ist bis zu einem gewissen Grade subjektiv. Davon abgesehen stellt sich auch immer die Frage, was zuerst bestimmt werden soll: Die Definition oder das For- 
schungsobjekt selbst? So entsteht ein Paradoxon, denn ,the relationship between definition and identification is circular: in order to establish a definition, you have to have a reliable set of representative examples, and these must therefore been identified first" (Wray 2002: 19).

Wie bereits erwähnt, gibt es trotz der terminologischen Uneinheitlichkeit einige Merkmale, die vielen Definitionen formelhafter Äußerungen gemeinsam sind, jedoch ist die Ganzheitlichkeit, wie es scheint, das entscheidende Merkmal. Da damit die psycholinguistische Perspektive ${ }^{2}$ in den Vordergrund tritt, kann hier als erstes der von Alison Wray verwendete Begriff „formulaic sequence“ („formelhafte Sequenz") behandelt werden (Wray 2002: 9). So ist eine formelhafte Sequenz „a sequence, continuous or discontinuous, of words or other elements, which is, or appears to be, prefabricated: that is, stored and retrieved whole from memory at the time of use, rather than being subject to generation or analysis by the language grammar" (ebd.).

Hier ist zu betonen, dass diese Definition auch in Wrays früheren Veröffentlichungen zu finden ist, aber in etwas anderer Formulierung, nämlich: „a sequence, continuous or discontinuous, of words or other meaning elements..." (z.B. Wray 2000: 455, Wray/Perkins 2000; Hervorhebung in der Definition - M.C.). Das Weglassen von meaning in Wray 2002 und in den späteren Arbeiten begründet die Autorin damit, dass die Formulierung „meaning elements“ diese Äußerungen / Wörter der Kindersprache oder die Sprache der Aphasiker nicht berücksichtigt, die als „customarily considered to be formulaic“, aber ,not decomposable into meaning elements" angesehen werden (Wray 2002: 283, Anm. 6). So ist in der neuen Definition eine wesentlich veränderte Auffassung der Formelhaftigkeit mit enthalten: Vorgeschlagen wird, dass ebenfalls eine Lautsequenz (aus einem Einzelmorphem bestehend) oder eine bedeutungslose Äußerung als formelhaft bezeichnet werden kann.

Die Autorin verweist bei der Begründung ihrer Begriffswahl darauf, dass damit weder die psycholinguistische Perspektive noch der soziale Kontext ausgeklammert werden: ,The word formulaic carries with it some associations of 'unity' and of 'custom' and 'habit', while sequence indicates that there is more than one discernible internal unit, of whatever kind" (Wray 2002: 9).

Für die Bezeichnung formelhafter Äußerungen werden auch andere Termini verwendet, die meisten von ihnen sind jedoch im Laufe der Zeit mit Assoziationen sehr unterschiedlicher Art beladen worden.

Beispielsweise ist der Begriff „Phraseologismus“ (als Bezeichnung einer festen Wortverbindung) im deutschsprachigen Bereich weit verbreitet; daher könnte er hinsichtlich der Zielsetzung dieser Arbeit auf den ersten Blick als relevant betrachtet werden. Da „Phraseologismus“ in der linguistischen Tradition mit der

${ }^{2} \mathrm{Da}$ sich die Psycholinguistik „mit mentalen Prozessen der Informationsverarbeitung [...] speziell in Bezug auf die Sprachverarbeitung beschäftigt" (Höhle 2010: 13), wird mit psycholinguistischer Perspektive eben diese Sichtweise gemeint. 
Phraseologie - als Teildisziplin der Linguistik - streng verknüpft ist, scheint der Begriff wenig geeignet, die psycholinguistischen Aspekte sprachlicher Einheiten hinreichend zu erfassen.

An dieser Stelle muss auf das spezifische Verhältnis von ,phraseologisch“ und „formelhaft" hingewiesen werden. Stephan Stein merkt dazu an (Stein 1995: 17): „Die Phraseologieforschung lässt sich denn auch als linguistische Bezugsdisziplin verstehen, bei der eine Analyse formelhaften Sprachgebrauchs ansetzen kann, doch formelhafte Sprache weist über die Grenzen der Phraseologie hinaus" (Hervorhebung - M.C.).

Darüber hinaus ist der Terminus „Phraseologismus“ zu vieldeutig. Von einigen Autoren wird zwischen Phraseologismen im engeren Sinne und Phraseologismen im weiteren Sinne unterschieden (z.B. Pilz 1981: 21 ff., Palm 1995: 1 ff., Fleischer 1997: 68 f.). Phraseologismen im engeren Sinne sind durch das Merkmal der Idiomatizität gekennzeichnet; im weiteren Sinne können unter dem Begriff auch Kollokationen, Sprichwörter und formelhafte Texte gemeint sein (vgl. Burger/Dobrovol'skij/Kühn/Norrick 2007: 2).

Die „Unklarheit“ des Begriffes „Phraseologismus“ zeigt die von Harald Burger dargestellte Basisklassifikation und -terminologie, die der Autor für die Gliederung des Gesamtbereichs der Phraseologie verwendet (Burger 2010: 36 ff.). Burgers Systematisierung zeigt einerseits, wie vieldeutig der Begriff „Phraseologismus" in der germanistischen Forschung ist. Andererseits lässt sich anhand der Klassifikation demonstrieren, dass auch andere Begriffe (z.B. „Kollokationen“, „Routineformeln“ oder „feste Phrasen“) in Anbetracht der Zielsetzung der vorliegenden Arbeit nicht geeignet sind.

Die „Kollokation“ kann - wie hier bei Burger - für den ganzen Bereich der nicht- bzw. schwach idiomatischen Phraseologismen verwendet werden (Burger 2010: 38). Darüber hinaus ist der Terminus „Kollokation“, der aus der angelsächsischen Tradition herkommt, auch zu sehr mit unterschiedlichen Bedeutungen vorbelastet. Die englische Phraseologieforschung unterscheidet zwischen „open collocation" („offenen Kollokationen“ - frei kombinierbaren Wortverbindungen, z.B white wine, to run a car), ,restricted collocations“ (,,restringierten Kollokationen" - Wortverbindungen mit einer teilidiomatisierten Konstituente, was einen Austausch ausschließt, z.B. white flag, to run a farm) und ,idioms“ (z.B. white lie, to run one's head against a brick wall - vgl. Gläser 1986: $38 \mathrm{ff}$ ); darunter gibt es „pure idioms“, bei denen sich die Gesamtbedeutung nicht aus den einzelnen Konstituentenbedeutungen ergibt (z.B. blow the gaff) und "figurative idioms“, die sowohl eine übertragene als auch eine wörtliche Bedeutung haben (z.B. to blow one's own trumpet) - vgl. z.B. Cowie 1998: 6 f.; in der deutschen Forschungsliteratur dagegen schließt der Begriff der Kollokation grundsätzlich das Idiomatische aus. ${ }^{3}$

${ }^{3}$ Kollokationen werden sowohl in einem weiteren Sinne verstanden (wie z.B. von Feilke 1996: 115 ff.) als auch in einem engeren - Franz Joseph Hausmann betrachtet nur Zwei-Wort-Verbindungen als Kollokationen (Hausmann 1984: 119). 
Burgers Darstellung ist zu entnehmen, dass der Begriff „feste Phrasen“ ebenfalls als zu eng betrachtet werden muss, da er sich nur auf einen Teil der Wortverbindungen, die sich durch ihre Ganzheitlichkeit / Formelhaftigkeit auszeichnen, bezieht.

Zu wenig umfassend ist auch der Terminus „Routineformel“. „Routineformeln" werden generell im Rahmen ihrer spezifischen Funktionen innerhalb der Kommunikation definiert und ,ihre Semantik ist zugunsten der Wahrnehmung der Funktionen häufig weitgehend außer Kraft gesetzt" (Stein 1995: 47). Demnach wird der Begriff „Routineformeln“ für die Bezeichnung nur einer Klasse von formelhaften Äußerungen verwendet (vgl. Coulmas 1981: 69, Gläser 1986: 49, Burger 2010: 36). Darüber hinaus wird der Terminus „Routineformel“ meist als Synonym für ,pragmatisches Idiom“ (Burger 1973: 58), „pragmatischer Phraseologismus“ (Burger/Buhofer/Sialm 1982: 105, Beckmann/König 2002: 421), „pragmatische Prägung“ (Feilke 1996: 214), kommunikative Formel“ (Fleischer 1997: 125), oder „pragmatische Phraseme“(Lüger 2007: 444).

Bei den angelsächsischen Arbeiten ist es jedoch nicht der Fall: Der Begriff prefabricated routine wird generell im breiteren Kontext, d.h. für die Bezeichnung der als Ganzes gespeicherten Äußerungen verwendet $-\mathrm{s}$. die Definitionen von Brown (1973: 87), Hakuta (1974: 289), Bolinger (1976: 1), Krashen/Scarcella (1978: 283).

Ebenfalls nicht angebracht sind Begriffe „linguistic routines“, „formulae“, „formulas“, „,set phrases“ (auch „gambit"). Da bei ihnen in erster Linie der interaktionistische Aspekt hervorgehoben wird, sind sie nicht zufrieden stellend. Die Verwendung des Begriffes "formula" wird von Norbert Schmitt und Ronald Carter so kommentiert: „The term formula is often used, but usually to mean a string of formulaic language with idiosyncratic conditions of use [...]" (Schmitt/Carter 2004: 4). Die Bezeichnungen wie „expressions“, „items“, „forms“ sind dagegen zu allgemein und erscheinen zu wenig präzise im psycholinguistischen Kontext.

Die Begriffe wie „,estgeprägte Sätze“, „lexical phrases“, „lexicalized sentence stems" sind irreführend, denn sie können jeweils als ,grammatische" Einheiten, die eine grammatische Verarbeitung voraussetzen, angesehen werden (vgl. Krishnamurthy 2002: 289); dies hat zur Folge, dass die psycholinguistische Perspektive dabei ganz verloren geht (wobei sie hingegen bei dem Begriff „chunks“ zu sehr dominiert).

Wie die oben gemachten Ausführungen zeigen, besteht in der angelsächsischen und deutschen Forschungsliteratur keine Einigkeit darüber, was für ein Oberbegriff für die Bezeichnung einer festen sprachlichen Einheit eingesetzt werden kann. Dieses Problem wird auch in Burger/Dobrovol'skij/Kühn/Norrick (2007: 2 ff.) - allerdings hauptsächlich in Bezug auf die Phraseologie - breit diskutiert. Als internationalen Terminus zur Bezeichnung einer phraseologischen Einheit schlagen die Autoren einen Begriff „Phrasem“ vor, dabei weisen sie jedoch darauf hin, dass der Begriff zu sehr mit anderen Deutungen vorbelastet ist (ebd.: 3): 
Der Terminus „Phrasem“ hat allerdings den Nachteil, dass durch das Suffix -em stark der Systemaspekt betont wird (vgl. „Phonem“, „Morphem“, „Lexem“,,Textem“). Es ist wohl nicht möglich, damit alles Formelhafte außerhalb der Idiome, Kollokationen usw. abzudecken. Sobald der engere Bereich der Phraseologie verlassen wird und Weiterungen in irgendeiner Richtung stattfinden, ist „Phrasem“ nur noch mit Einschränkungen brauchbar. Die Herausgeber schlagen vor, „Phrasem“/,,phraseme“ - dort, wo der Terminus gebraucht wird - für den engeren Bereich der Phraseologie (also als Oberbegriff für Idiome und Kollokationen) zu verwenden, bei einer weiten Konzeption aber auf andere Termini als Oberbegriffe auszuweichen (z.B. „formelhafte Sprache“, ,formulaic language“ [...]).

\section{Identifizierung formelhafter Äußerungen}

Alle vorgenannten Probleme mit der Begriffsbestimmung haben zur Folge, dass die Identifizierung formelhafter Äußerungen eine rechte Herausforderung darstellt. Häufigkeit des Auftretens und Ganzheitlichkeit sind die Merkmale, die bei der Identifizierung formelhafter Äußerungen im Text oder in der gesprochenen Sprache behilflich sein können.

\subsection{Häufigkeit als Hilfe bei der Identifizierung}

An dieser Stelle ist auf Folgendes aufmerksam zu machen: Das Verhältnis zwischen Formelhaftigkeit und Häufigkeit ist äußerst kompliziert. Erstens müssen nicht alle Konstruktionen, die in der gesprochenen Sprache oder in einem Text häufig auftreten, ganzheitlich gespeichert werden, zweitens gibt es Äußerungen, die selten anzutreffen sind, die jedoch bestimmt als formelhaft betrachtet werden können (s. Nesi/Basturkmen 2006: 286).

Wie bereits erwähnt ist es nicht einfach zu entscheiden, ob eine Äußerung aus dem Gedächtnis als Ganzes abgerufen wird oder nicht. Zwar ermöglichen Computerprogramme die Analyse von großen Textkorpora auf Häufigkeit, doch die auf diese Weise gewonnenen Daten erlauben es nicht, eine präzise Differenzierung „formelhaft oder nicht" zu machen: Da eine solche Untersuchung darauf abzielt, regelmäßige Verknüpfungen bestimmter sprachlicher Elemente herauszuarbeiten, können anhand einer Korpusanalyse lediglich ganz feste Äußerungen oder sehr häufig auftretende Ausdrücke identifiziert werden. Foster (2001: 81) merkt folgendes an: "Even a corpus as large as The Bank of English at the University of Birmingham, now nearly three hundred million words, fails to show even a single example of many phrases that would be considered a normal part of any native speaker's repertoire".

Dies weist nach Fosters Auffassung darauf hin, dass die „Sammlung“ der Äußerungen, die von einem Sprecher ganzheitlich memoriert worden sind, sehr viele Elemente enthält, die in einem Sprachkorpus überhaupt nicht häufig auftreten müssen. 
Die computergestützte Analyse der Textkorpora auf Häufigkeit wirft andere Probleme auf. Erstens müssen Ad-hoc-Entscheidungen darüber getroffen werden, aus wie vielen Worten sich die gesuchte Äußerung zusammensetzen soll, und welche demnach als ,häufig“ gelten kann. Dabei ist zu berücksichtigen, dass die Häufigkeit einer immer wiederkehrenden Äußerung umgekehrt proportional zu ihrer Länge ist (s. De Cock/Granger/Leech/McEnery 1998: 71), was weitere Probleme nach sich zieht.

Besondere Schwierigkeiten bereiten formelhafte Konstruktionen, die keine feste Struktur haben. Es sind Formen mit offenen slots, d.h. mit Lücken für Elemente aus anderen Wortklassen (z.B. how are you + Zeitangabe, z.B. how are you this morning, vgl. Pawley/Syder 1983: 210). Das Fehlen einer festen Struktur ist übrigens der Grund dafür, dass diese „formulaic frames with analyzed slots“ (vgl. Peters 1983: 4) in der Forschung häufig übersehen wurden, da andere Merkmale, z.B. Idiomatizität, als Voraussetzung für die ganzheitliche Speicherung gesehen wurde (vgl. Conrad/Biber 2004: 69).

In Korpora der gesprochenen Sprache findet sich beispielsweise die Transkription von Interjektionen (,aha“, „nanu“, ,ach“, ,ja“ usw.), so muss der Forscher darüber entscheiden, ob (und evtl. wann) sie als Wörter zu betrachten sind. Alison Wray weist mit Recht darauf hin, dass solche Ad-hoc-Entscheidungen ein subjektives Urteil darstellen (Wray 2002: 27). Wenn hinzukommt, dass bereits die Auswahl der Korpora subjektiv ist, wird Folgendes ersichtlich: Computeranalyse der Textkorpora auf Häufigkeit kann auf keinen Fall als absolut objektiv gelten.

\subsection{Ganzheitlichkeit als Hilfe bei der Identifizierung}

Ein maßgebliches Merkmal formelhafter Äußerungen ist ihre Festigkeit, allerdings muss an dieser Stelle auf Folgendes hingewiesen werden: Es gibt keine objektiven Kriterien, um darüber zu entscheiden, ob eine Äußerung fest, d.h. im Gedächtnis als Ganzes gespeichert worden ist (z.B. Bahns/Burmeister/Vogel 1986, Wray 2002).

Weil man in der Forschung davon ausgeht, dass formelhafte Äußerungen aus dem Gedächtnis als Ganzes abgerufen werden, wird angenommen, dass sie flüssiger produziert werden als die nach Regeln gebildeten Äußerungen. Als wichtiger Hinweis für die Ganzheitlichkeit der Speicherung kann demnach die phonologische Kohärenz einer Äußerung betrachtet werden, d.h. flüssige und zögerungsfreie Produktion ohne Planungspausen in der Intonationskurve (selbstverständlich kann sich dieses Kriterium ausschließlich auf die gesprochene Sprache beziehen).

Manfred Raupach (1984: 114) stellt die These auf, dass Pausen und Verzögerungen in der Sprachproduktion an den Grenzen formelhafter Äußerungen eintreten, was nach seiner Auffassung bei der Identifizierung formelhafter Äußerungen hilfreich sein kann: ,, a formal approach to identifying formula units in spontaneous 
speech must, as a first step, list the strings which are not interrupted by unfilled pauses" (ebd.: 116).

Wortakzent und Artikulation stehen auch in Verbindung zu dem Problem phonologischer Kohärenz formelhafter Äußerungen. Bereits Jespersen (1924: 18) sah den Wortakzent als ihr definierendes Merkmal, indem er argumentierte, es sei eigentlich nicht möglich, innerhalb einer formelhaften Äußerung den Akzent zu ändern oder eine Pause zu machen (ebd.): ,[...] you cannot even change the stress, saying "How do you do?" or make a pause between the words [...]. Even though it may still be possible, after saying "How do you do?" in the usual way to some of the people present, to alter the stress and say "And how do you do, little Mary?", the phrase is for all practical purposes one unchanged and unchangeable formula".

Dies stimmt mit der Beobachtung überein, dass die Artikulation innerhalb einer formelhaften Äußerung häufig unpräzise sein kann (vgl. van Lancker et. al 1981, Brown 1990), denn „the strongest artikulation occurs on stressed syllables“ (Wray 2002: 38).

Im Rahmen der Festigkeit soll die sog. semantische Prosodie gesehen werden - eine Eigenschaft, die für manche formelhafte Äußerungen charakteristisch ist. Der Begriff „semantische Prosodie“ wurde durch die Korpuslinguistik eingeführt und bedeutet die Tatsache, dass einige Wörter eine positive oder eine negative Konnotation haben können, was von dem Kontext und von der Bedeutung eines Wortes abhängt. Von Bill Louw wird semantische Prosodie definiert als ,the consistent aura of meaning with which a form is imbued by its collocates" (Louw 1993: 157), von Sinclair hingegen als: "[...] an aura of meaning that is subliminal, in that we only become aware of it when we see a large number of typical instances all together" (Sinclair 2004:18). ${ }^{4}$ Von der positiven semantischen Prosodie kann gesprochen werden, wenn das Kernwort ausschließlich mit positiven semantischen Eigenschaften (z.B. provide), von der positiven semantischen Prosodie dagegen, wenn das Kernwort ausschließlich mit negativen semantischen Eigenschaften konnotiert (z.B. cause).

Das Phänomen der semantischen Prosodie bezüglich der formelhaften Äußerungen verdeutlicht das von Schmitt/Carter (2004:8) präsentierte Beispiel:

Male chauvinism was rife in medicine in those days.

Fears are now rife that the price could plunge well below 30p by the end of the year.

Proficient language users know that rife is used to express the meaning 'something undesirable is too common', and that the formulaic sequence in which rife is embedded typically has the following structure:

SOMETHING UNDESIRABLE is/are rife in LOCATION/TIME

${ }^{4}$ Stubbs (1995) beschreibt dasselbe Phänomen, indem er den Begriff collocational prosody verwendet. 
Die Autoren machen jedoch darauf aufmerksam, dass das Phänomen der semantischen Prosodie bei formelhaften Äußerungen noch eine offene Frage ist (ebd.: 9). Bestimmt kann dieser Aspekt bei der Identifizierung formelhafter ÄuBerungen hilfreich sein: Es gibt sehr starke empirische Beweise dafür, dass die semantische Prosodie des Verbs bei Kollokationen (die einen bestimmten Typ formelhafter Äußerungen darstellen) automatisch und sehr schnell "erkannt" wird (Ellis/Frey 2009: 493).

Da die oben erwähnten Methoden so viele Probleme aufwerfen, greifen Forscher häufig auf eine andere zurück, um formelhafte/ganzheitliche Äußerungen zu identifizieren, nämlich auf die Intuition. Aus wissenschaftlicher Perspektive ist die Intuition zwar kein einwandfreies Kriterium, über die Formelhaftigkeit zu urteilen, es gibt jedoch Forscher, die meinen, sprachbewusste und qualifizierte Muttersprachler seien für die Identifizierungstätigkeit geeigneter als Computerprogramme (vgl. Aguado 2002: 46); andere behaupten ferner, dass intuitive Beurteilungen im Hinblick auf die Identifizierung formelhafter Äußerungen im Einklang mit wissenschaftlichen Ergebnissen stehen (Nattinger / DeCarrico 1992: 28).

Demnach wird die Intuition häufig von der Forschung herangezogen und beim Aufbau der Experimente, bei der Auswahl der Beispiele und Interpretation der Ergebnisse berücksichtigt (z.B. Bahns / Burmeister / Vogel 1986: 700, Erman / Warren 2000, Foster 2001: 81).

Natürlicherweise ist die Anwendung der Intuition auch mit vielfältigen Problemen verbunden. Beispielsweise berichten Foster / Skehan (1996), dass die Muttersprachler, die die Transkripte auf „Vorfabriziertheit“ hin beurteilen sollten, sich über Konzentrationsschwierigkeiten und Schwierigkeiten in Bezug auf die Grenzziehung zwischen formelhaften und nicht formalhaften Äußerungen beklagt haben. Darüber hinaus wird in der Literatur hervorgehoben, dass die Anwendung der Intuition den Forscher vor weitere Probleme stellt (Wray 2009: 39 ff.).

\section{Schlussbemerkungen}

Es ist im Kapitel 2 bereits erwähnt worden, dass der Forscher häufig darüber entscheiden muss, ob zuerst die Definition oder das Forschungsobjekt bestimmt werden soll, was zu einer paradoxen Situation führt. Dies ist der Fall bei formelhaften Äußerungen - ihre terminologische Uneinheitlichkeit und konzeptuelle Unschärfe (die natürlicherweise wechselseitig bedingt sind) haben ein wissenschaftliches Chaos zur Folge und wirken sich negativ auf die Forschungssituation auf diesem Gebiet aus. Aber aus diesem Paradoxon gibt es einen Ausweg: Zuerst können einige prototypische „Exemplare“ identifiziert werden; mit ihrer Hilfe soll die Kriterienbestimmung vorgenommen werden (die später auch auf die weniger prototypischen Exemplare angewendet werden kann - vgl. Wray 2002: 284). Diese Vorgehensweise erleichtert die Identifizierung; dabei können verschiedene Me- 
thoden gleichzeitig eingesetzt werden. Darüber hinaus muss man die Intuition nicht ausschließen: In der Forschung wird darauf verwiesen, dass die Anwendung der Intuition - mit Beibehaltung einiger Bestimmungen - sogar als wissenschaftlich akzeptabel gelten kann (Read/Nation 2004: 29). Um dies zu gewährleisten, ist es in erster Linie erforderlich, den Urteilern genau zu erläutern, was mit der Formelhaftigkeit / Ganzheitlichkeit einer Äußerung gemeint wird; diese Frage soll früher eingehend diskutiert werden. Außerdem kann vorher angenommen werden, dass mehrere (z.B. vier oder fünf) Urteiler, welche über die Formelhaftigkeit oder Nicht-Formelhaftigkeit einer Äußerung bestimmen, zu dem gleichen Ergebnis kommen müssen, bevor eine Entscheidung fällt (ebd.: 29 f.).

Allerdings ist bei empirischen Untersuchungen auf diesem Gebiet zu beachten, dass die Ergebnisse mit entsprechender Vorsicht zu interpretieren sind, denn Formelhaftigkeit ist „like the elephant differently described by blind men with access to different parts of ist huge mass. [...] the point of the metaphor is that the blind men don't know if they are in fact describing aspects of the same thing, because they can't see the elephant. [...] But the elephant in the room is that we don't know if there is just one elephant in the room" (Wray 2012: 239).

\section{Literatur}

Aguado, Karin: Imitation als Erwerbsstrategie. Interaktive und kognitive Dimensionen des Fremdsprachenerwerbs (Habilitationsschrift). Bielefeld 2002.

Bahns, Jens / Burmeister, Hartmut / Vogel, Thomas: The pragmatics of formulas in L2 learner speech: use and development. In: Journal of Pragmatics 10 (6) 1986. S. 693-723.

Beckmann, Susanne / König, Peter-Paul: Pragmatische Phraseologismen. In: Cruse, D. Alan / Hundsnurscher, Franz / Job, Michael / Lutzeier, Peter R. (Hrsg.): Lexikologie. Lexicology. Ein internationales Handbuch zur Natur und Struktur von Wörtern und Wortschätzen. Berlin, New York 2002. S. 421-428.

Bolinger, Dwight: Meaning and memory. In: Forum Linguisticum 1, 1976. S. 1-14.

Brown, Gillian: Listening to spoken English. London, New York 1990.

Brown, Roger: A first language: The early stages. London 1973.

Burger, Harald: Idiomatik des Deutschen. Tübingen 1973.

Burger, Harald: Phraseologie: eine Einführung am Beispiel des Deutschen. Berlin 2010.

Burger, Harald / Buhofer, Annelies / Sialm, Ambros: Handbuch der Phraseologie. Berlin, New York 1982.

Burger, Harald / Dobrovol'skij, Dmitrij / Kühn,Peter / Norrick, Neal: Einführung / Subject area, terminology and research topics. In: Burger, Harald / Dobrovol'skij, Dmitrij / Kühn,Peter / Norrick, Neal (Hrsg.): Phraseologie. Ein internationales Handbuch zeitgenössischer Forschung. Bd. 1. Berlin 2007. S. 1-10.

Conrad, Susan / Biber, Douglas: The frequency and use of lexical bundles in conversation and academic prose. In: Lexicographica: International Annual for Lexicography 20, 2004. S. 56-71.

Coulmas, Florian: Routine im Gespräch: zur pragmatischen Fundierung der Idiomatik. Wiesbaden 1981.

Cowie, Anthony P.: Phraseology. Theory, Analysis, and Applications. Oxford 1998.

Czarnecka, Małgorzata: Formelhaft oder nicht? Die wichtigsten Merkmale der formelhaften Sequenzen. In: Glottodidactica XXXVI, 2010. S. 33-43. 
Czarnecka, Małgorzata: Formelhafte Sequenzen in der Erst- und Zweitsprache: Versuch einer Begriffsbestimmung aus psycholinguistischer Perspektive. In: Germanica Wratislaviensia 133, 2011, S. 189-199.

De Cock, Sylvie / Granger, Sylviane / Leech, Geoffrey / McEnery, Tony: An automated approach to the phrasicon of EFL learners. In :Granger, Sylviane (ed.): Learner English on Computer. London and New York 1998. S. 67-79.

Ellis, Nick C. / Frey, Eric: The psycholinguistic reality of collocation and semantic prosody (2): Affective priming. In: Corrigan, Roberta / Moravcsik, Edith A. / Ouali, Hamid / Wheatley, Kathleen M. (Hrsg.): Formulaic Language. Volume 2: Acquisition, loss, psychological reality, and functional explanations. Amsterdam, Philadelphia 2009. S. 473-497.

Erman, Britt / Warren, Beatrice: The idiom principle and the open choice principle. In: Text 20 (1), 2000. S. 29-62.

Feilke, Helmuth: Sprache als soziale Gestalt. Frankfurt am Main 1996.

Fischer, Karin / Stefanowitsch, Anatol: Konstruktionsgrammatik. Ein Überblick. In: Fischer, Karin / Stefanowitsch, Anatol (Hrsg.): Konstruktionsgrammatik. Von der Anwendung zur Theorie. Tübingen 2006. S. 19-38.

Fleischer, Wolfgang: Phraseologie der deutschen Gegenwartssprache. 2. durchgesehene und ergänzte Auflage. Tübingen 1997.

Foster, Pauline: Rules and routines: A consideration of their role in task-based language production of native and non-native speakers. In: Bygate, Martin / Skehan, Peter / Swain, Merrill (Hrsg.): Reseaching pedagogic tasks: Second language learning, teaching and testing. New York 2001. S. 75-93.

Foster, Pauline / Skehan, Peter: The influence of planning and task type on second langauge performances. In: Studies in Second Language Acquisition, 18 (3), 1996. S. 299-324.

Gläser, Rosemarie: Phraseologie der englischen Sprache. Tübingen 1986.

Hakuta, Kenji: Prefabricated patterns and the emergence of structure in second language acquisition. In: Language Learning 24, 1974. S. 287-298.

Hausmann, Franz Josef: Wortschatzlernen ist Kollokationslernen. Zum Lehren und Lernen französischer Wortverbindungen. In: Praxis des neusprachlichen Unterrichts 31, 1984. S. 395-406.

Höhle, Barbara (Hrsg.): Psycholinguistik. Berlin 2010.

Jespersen, Otto: The Philosophy of Grammar. London 1924.

Krishnamurthy Ramesh: Language as Chunks, not Words. In: JALT-Publications (online), Shizuoka $2002 \mathrm{http}: / /$ jalt-publications.org/archive/proceedings/2002/288.pdf [Zugriff am 26.03.2013]

Krashen, Stephen D. / Scarcella, Robin: On routines and patterns in language acquisition and performance. In: Language Learning 28, 1978. S. 283-300.

Leiss, Elisabeth: Konstruktionsgrammatik versus Universalgrammatik. In: Schmöe, Friederike / Glück, Helmut (Hrsg.): Wie wir sprechen und schreiben: Festschrift für Helmut Glück zum 60. Geburtstag. Wiesbaden 2009. S. 17-28.

Louw, Bill: Irony in the text or insincerity in the writer? The diagnostic potential of semantic prosodies. In: Baker, Mona / Francis, Gill / Tognini-Bonelli, Elena (Hrsg.): Text and technology. Amsterdam, Philadelphia 1993. S. 157-176.

Lüger, Heinz-Helmut: Pragmatische Phraseme: Routineformeln. In: Burger, Harald / Dobrovol'skij, Dmitrij / Kühn, Peter / Norrick, Neal (Hrsg.): Phraseologie. Ein internationales Handbuch der zeitgenössischen Forschung. 1. Halbband. Berlin, New York 2007. S. 444-459.

Nattinger, James R. / DeCarrico, Jeanette S.: Lexical phrases and language teaching. New York 1992.

Nesi, Hilary / Basturkmen, Helen: Lexical bundles and discourse signalling in academic lectures. In: International Journal of Corpus Linguistics 11, 2006. S. 147-168.

Palm, Christine: Phraseologie: eine Einführung. Tübingen 1995.

Pawley, Andrew / Syder, Frances Hodgetts: Two puzzles for linguistic theory: nativelike selection and nativelike fluency. In: Richards, Jack C./ Schmidt, Richard W. (Hrsg.): Language and Communication. London 1983. S. 191-226.

Germanica Wratislaviensia 140, 2016

(C) for this edition by CNS 
Peters, Ann M.: The units of language acquisition. Cambridge 1983.

Pilz, Klaus Dieter: Phraseologie: Redensartenforschung. Stuttgart 1981.

Raupach, Manfred: Formulae in second language speech production. In: Dechert, Hans W. I Möhle, Dorothea / Raupach, Manfred (Hrsg.): Second language production. Tübingen 1984. S. 114-137.

Read, John / Nation, Paul: Measurement of formulaic sequences. In: Schmitt, Norbert (Hrsg.): Formulaic Sequences. Amsterdam 2004. S. 23-35.

Roche, Jörg / Reher, Janina / Simic, Mirjana: Focus on Handlung: Zum Konzept des handlungsorientierten Erwerbs sprachlicher, sozialer und demokratischer Kompetenzen im Rahmen einer Kinder-Akademie. Münster 2012.

Schmitt, Norbert / Carter, Ronald: Formulaic sequences in action. An introduction. In: Schmitt, Norbert (Hrsg.): Formulaic sequences: acquisition, processing, and use. Amsterdam 2004. S. 1-22.

Sinclair, John M.: Trust the text. Language, Corpus and Discourse. London 2004.

Stein, Stephan: Formelhafte Sprache. Frankfurt am Main 1995.

Stubbs, Michael: Collocations and semantic profiles: on the cause of the trouble with quantitative studies. In: Functions of Language 2 (1), 1995. S. 23-55.

Stubbs, Michael: Words and Phrases: Corpus Studies of Lexical Semantics. Oxford 2001.

Tomasello, Michael: Konstruktionsgrammatik und früher Erstspracherwerb. In: Fischer, Karin / Stefanowitsch, Anatol (Hrsg.): Konstruktionsgrammatik. Tübingen. S. 19-38.

Van Lancker, Diana / Canter, Gerald J. / Terbeek, Dale: Disambiguation of ditropic sentences: Acoustic and phonetic cues. In: Journal of Speech and Hearing Research, 24, 1981. S. 330-335.

Wray, Alison: Formulaic sequences in second language teaching: Principle and practice. Applied Linguistic 21 (4), 2000. S. 463-489.

Wray, Alison: Formulaic Language and the Lexicon. Cambridge 2002.

Wray, Alison: Identifying formulaic language: Persistent challenges and new opportunities. In: Corrigan, Roberta / Moravcsik, Edith A. / Ouali, Hamid / Wheatley, Kathleen M. (Hrsg.): Formulaic Language. Volume 1: Distribution and historical change. Amsterdam, Philadelphia 2009. S. 27-52.

Wray, Alison: What Do We (Think We) Know About Formulaic Language? An Evaluation of the Current State of Play. In: Annual Review of Applied Linguistics 32, 2012. S. 231-254.

Wray, Alison / Perkins, Michael R.: The functions of formulaic language: An integrated model. In: Language \& Communication 20 (1), 2000. S. 1-28.

\section{Abstracts}

Der Begriff formelhafter Äußerungen umfasst unterschiedliche Arten von Wortverbindungen, die vom Gedächtnis als Ganzes gespeichert und abgerufen werden. Der Beitrag diskutiert zwei in diesem Zusammenhang zentrale Fragen: Wie können formelhafte Äußerungen definiert und identifiziert werden. Formelhafte Äußerungen sind nämlich so verschiedenartig, dass es schwierig ist, ihre Definition zu formulieren und ihre Merkmale zu beschreiben. Die derzeit existierenden Methoden der Identifizierung (z.B. aufgrund von Häufigkeit) werden kritisiert, denn alle haben ihre Nachteile; die Anwendung der Intuition, die häufig von der Forschung herangezogen wird, ist auch mit vielfältigen Problemen verbunden.

Schlüsselwörter: formelhafte Äußerungen, Definition, Identifizierung, Merkmale 


\title{
"How many elephants?" - Problems with defining and identifying formulaic sequences
}

The term "formulaic sequences" encompasses various types of word strings which appear to be stored and retrieved as holistic units from the memory. This article discusses two major problems in the study of formulaic sequences: how to define their features and how to identify these sequences in discourse. The problem is-that formulaic sequences exist in so many forms that it is difficult to develop a definition of this phenomenon and to find the main characteristics of formulaic sequences. The existing methods of identifying formulaic sequences (e.g. by frequency in the corpus) can be used to some degree but each of them has its drawbacks. Even drawing on the individual's intuition as the basis for identifying these sequences runs into its own serious problems.

Keywords: formulaic sequences, definition, identifying, characteristics

\author{
Małgorzata Czarnecka \\ Uniwersytet Wrocławski \\ Instytut Filologii Germańskiej \\ pl. Biskupa Nankiera 15b \\ 50-140 Wrocław \\ Polen \\ E-Mail: malgorzataczarnecka4@gmail.com
}

